\title{
Influence of Pacing by Periodic Auditory Stimuli on Movement Continuation: Comparison with Self-regulated Periodic Movement
}

\author{
Masanori Ito, PT, MS ${ }^{1,3)^{*}}$, Naoki Kado, PT, PhD ${ }^{1)}$, Toshiaki Suzuki, PT, DMSc ${ }^{2)}$, \\ Hiroshi Ando, MD, $\mathrm{PhD}^{3)}$ \\ 1) Department of Physical Therapy, Kobe College of Rehabilitation and Welfare: 1-2-2 Kominatodori, \\ Chuo-ku, Kobe-shi, Hyogo 650-0026, Japan. TEL: +81 78-361-2888, FAX: +81 78-361-2880 \\ 2) Clinical Physical Therapy Laboratory, Kansai University of Health Sciences \\ 3) Kobe University Graduate School of Health Sciences
}

\begin{abstract}
Purpose] The purpose of this study was to investigate the influence of external pacing with periodic auditory stimuli on the control of periodic movement. [Subjects and Methods] Eighteen healthy subjects performed self-paced, synchronization-continuation, and syncopation-continuation tapping. Inter-onset intervals were 1,000, 2,000 and $5,000 \mathrm{~ms}$. The variability of inter-tap intervals was compared between the different pacing conditions and between self-paced tapping and each continuation phase. [Results] There were no significant differences in the mean and standard deviation of the inter-tap interval between pacing conditions. For the 1,000 and 5,000 ms tasks, there were significant differences in the mean inter-tap interval following auditory pacing compared with self-pacing. For the 2,000 ms syncopation condition and 5,000 ms task, there were significant differences from self-pacing in the standard deviation of the inter-tap interval following auditory pacing. [Conclusion] These results suggest that the accuracy of periodic movement with intervals of 1,000 and 5,000 ms can be improved by the use of auditory pacing. However, the consistency of periodic movement is mainly dependent on the inherent skill of the individual; thus, improvement of consistency based on pacing is unlikely.

Key words: Pacing, Tapping, Auditory
\end{abstract}

(This article was submitted Mar. 19, 2013, and was accepted May 4, 2013)

\section{INTRODUCTION}

Producing rhythmic and periodic movement is an important aspect of movement control. For many years, there has been interest in whether or not rhythmic pacing has an effect on movement. Evidence suggesting that rhythmic pacing does affect movement comes from physical therapy studies. One such study found that during a task involving flexion and extension of the elbow, variability in the electromyogram patterns of the biceps brachii decreased with pacing supplied by a regular auditory rhythm ${ }^{1)}$. During physical therapy, external events such as auditory and visual stimuli might be used to facilitate periodic movement. Some individuals are able to perform rhythmic movement when coordinating the movement with external stimuli, but cannot continue the rhythmic movement without it.

A continuation paradigm of sensorimotor synchronization is one of the techniques used for evaluating the role of pacing by periodic external stimuli. In this paradigm, a subject taps in synchrony with a series of isochronous stimuli (synchronization phase) and continues to tap with the same pace once the external stimulus is removed (continuation

${ }^{*}$ To whom correspondence should be addressed.

E-mail: itou@sumire-academy.ac.jp phase). In previous studies using the synchronization-continuation paradigm, brain activity ${ }^{2-4)}$ and the variability of the inter-tap interval (ITI) ${ }^{5,6)}$ were examined, and a comparison was made between the continuation and synchronization phases. The comparison of these 2 phases indicated the accuracy of the timekeeper in the absence of an external pacing event. Such comparisons between the continuation and synchronization phases are important for understanding the effects of physical therapy using an external event as a trigger; however, movement should also be compared before and after external pacing. Before external pacing, movement is based on an individual's intrinsic motor control ability. It is important to compare a subject's intrinsic ability with their movement ability during the continuation phase, after the external pacing.

Using the continuation paradigm, several studies have investigated both synchronization and syncopation to assess pacing ${ }^{7,8)}$. To study syncopation, the subjects were asked to make their taps coincide with the midpoint of each stimulus in the external pacing sequence. Differences between syncopation and synchronization were described as follows ${ }^{9}$. First, since subjects were requested to tap halfway between stimulus pulses in a syncopation study, the effective waiting period was half that of the synchronization study. Second, while responses during synchronization usually preceded the stimulus in a truly anticipatory fashion, responses dur- 
ing syncopation contained a reactive component; tapping occurred in advance of the last stimulus and preceded the current stimulus by approximately the same amount of time. These features show that the deviations between taps and stimulus onset (asynchronies) clearly differ between synchronization and syncopation.

In physical therapy, activities involving both synchronization and syncopation are available, and the selection of activities is left to the judgment of each therapist. The purpose of this study was to compare the use of self-paced tapping, synchronization-continuation tapping, and syncopation-continuation tapping as external stimuli to control rhythmic movement.

\section{SUBJECTS AND METHODS}

Eighteen healthy subjects (10 women and 8 men; mean age, 23.9 years; age range, 18-37 years) participated in this study. None of the subjects had musical training. All were strongly right-handed according to the Edinburgh Handedness Inventory ${ }^{10)}$ (mean laterality quotient, 91.2; range, 66.6-100). Subjects had no motor function abnormalities of the right index finger and no hearing abnormalities at test frequencies of $1,000 \mathrm{~Hz}$ and $4,000 \mathrm{~Hz}$. Informed consent was obtained from all the subjects. This study was approved by the Ethics Committee of Kobe College of Rehabilitation and Welfare.

A telemetry EMG measuring system (MQ8; KISSEICOMTEC, Matsumoto City, Japan) was loaded onto a PC (NEC, VersaPro VY20F/AG-W). The auditory stimulus system was set up using Viking Quest (Nicolet Biomedical, Wisconsin, USA). The presented auditory stimulus and the precise moment of the tapping were recorded using a data acquisition system (VitalRecorder2, KISSEICOMTEC), and an A/D converter card (ADA16-32/2 [CB] F, CONTEC). Recorded signals were analyzed using an EMG signal analysis program with a video movie (BIMUTASVideo, KISSEICOMTEC).

The subjects were required to tap with the right index finger during the following 3 tasks. Task 1: self-paced tapping with no auditory stimuli; subjects made 15 successive taps for the directed interval. Task 2: synchronization-continuation tapping; subjects tapped in synchrony with 15 periodic auditory stimuli (pacing phase) and then continued to perform 15 taps at the same rate without the auditory stimuli (continuation phase). Task 3: syncopation-continuation tapping; subjects tapped in synchrony with the midpoint of each stimulus of 15 periodic auditory stimuli (pacing phase) and then continued to perform 15 taps at the same rate without the auditory stimuli (continuation phase). In tasks 2 and 3 , the subjects were instructed to start tapping at the third auditory stimulus. Each subject performed the 3 tasks over a period of 3 days. Task 1 was performed on day 1 , and tasks 2 and 3 were performed at random.

The auditory stimulus intensity was $65 \mathrm{~dB}$, its frequency was $750 \mathrm{~Hz}$, and its duration was $25 \mathrm{~ms}$. The time difference between the onset of two successive stimuli was defined as the inter-onset interval (IOI), and a total of three different IOIs were used in this study: $1,000 \mathrm{~ms}, 2,000 \mathrm{~ms}$ and 5,000 ms. These were also the directed intervals used in task 1. Auditory stimuli were delivered via headphones.

The experiment was conducted in a quiet room. The subjects were seated with the right forearm placed in a pronated position on a desk in front of them. The precise moment of the tapping with the right index finger was detected using a contact sensor that was attached to the distal end of the right index finger. The tapping was performed by flexion and extension of the metacarpophalangeal joint of the right index finger. The subjects were not allowed to time themselves by any other physical movement such as head nodding or foot tapping. Their eyes were closed to exclude the influence of vision during the tasks. For task 1, the experimenter indicated the movement interval to subjects by verbal instruction, and the subjects started tapping after the command "please begin tapping." Tasks 2 and 3 began after individuals received instructions for performing the task. All tasks were repeated 3 times for each IOI, giving a total of 9 trials that were performed at random by each subject.

ITI was defined as the time difference between successive taps. In all tasks, the latter 10 ITIs in each sequence were used for analysis. In task 1, the lowest standard deviation (SD) for the ITI in the 3 trials was selected as the representative data for each subject. In tasks 2 and 3, the lowest SD for the ITI in the pacing phase of the 3 trials was selected as the representative data for each subject. The paired t-test was used to compare the pacing phases of tasks 2 and 3. One-way analysis of variance (ANOVA) was used to compare task 1 and the continuation phases of tasks 2 and 3 . When a significant difference was recognized, paired comparisons were performed using Tukey's post hoc test. The autocorrelation functions were used for analysis of ITI time series data. The unpaired t-test and correlation analysis were used for additional analysis when necessary. $\mathrm{p}$ values $<0.05$ were considered statistically significant. Statistical analyses were performed using Excel-Toukei 2008 for Windows (Social Survey Research Information Co. Ltd., Tokyo, Japan).

\section{RESULTS}

The ITI series of task 1 for each subject are shown in Fig. 1. With a directed interval of $1,000 \mathrm{~ms}$, more than half of the subjects responded after an interval longer than the target of 1,000 ms. With a directed interval of 2,000 ms, there were few subjects who achieved the target interval of $2,000 \mathrm{~ms}$, with most subjects having a shorter or longer interval than the $2,000 \mathrm{~ms}$ target. Using a $5,000 \mathrm{~ms}$ interval, more than half of the subjects responded after an interval shorter than the 5,000 ms target.

The ITI series of tasks 2 and 3 are shown in Fig. 2. During the pacing phase, the ITI series of most subjects were close to the target duration; as the duration of the IOI increased, several subjects developed a wavy ITI series. During the continuation phases using an IOI of $1,000 \mathrm{~ms}$, one subject tended to respond with intervals longer than $1,000 \mathrm{~ms}$. With an IOI of 2,000 and 5,000 ms, drifts from the target interval increased compared to that of the $1,000 \mathrm{~ms}$ IOI; this difference was more marked for the syncopation condition. 

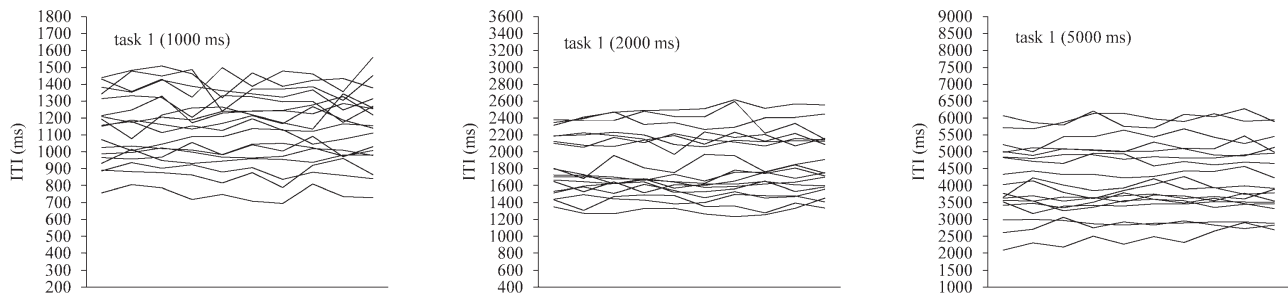

Fig. 1. Task 1 ITI series for each subject with directed intervals of $1,000 \mathrm{~ms}, 2,000 \mathrm{~ms}$ and 5,000 ms. Each ITI line is the plot of 10 ITI points.
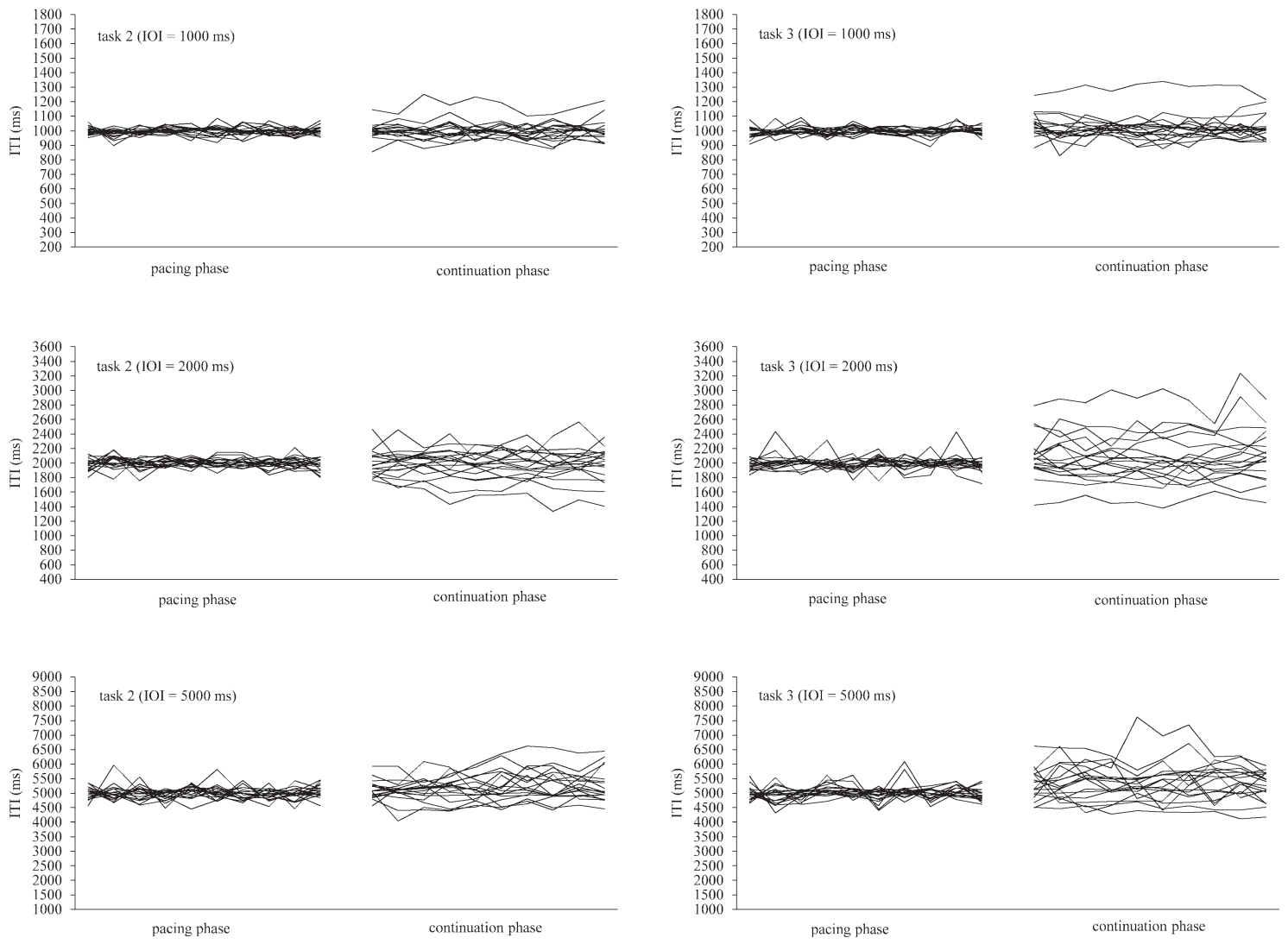

Fig. 2. Task 2 and 3 ITI series for each subject at IOIs of $1,000 \mathrm{~ms}, 2,000 \mathrm{~ms}$ and 5,000 ms. Each ITI line is the plot of 10 ITI points.

Table 1. ITIs in the pacing phase of tasks 2 and 3

\begin{tabular}{cccccc}
\hline & \multicolumn{2}{c}{ Mean $(\mathrm{ms})$} & & \multicolumn{2}{c}{$\mathrm{SD}(\mathrm{ms})$} \\
\cline { 2 - 3 } \cline { 5 - 6 } IOI $(\mathrm{ms})$ & task 2 & task 3 & & task 2 & task3 \\
\hline 1,000 & $996.3 \pm 3.7$ & $997.3 \pm 4.4$ & & $30.5 \pm 9.3$ & $29.7 \pm 12.9$ \\
2,000 & $1,999.2 \pm 10.9$ & $1,994.1 \pm 15.6$ & & $75.0 \pm 27.0$ & $83.0 \pm 51.2$ \\
5,000 & $5,003.4 \pm 19.9$ & $5,004.1 \pm 49.0$ & & $218.0 \pm 102.8$ & $221.8 \pm 121.7$ \\
\hline
\end{tabular}

Values are mean \pm SD, IOI: inter-onset interval

The mean and SD of the ITI of the pacing phases of tasks 2 and 3 are shown in Table 1. Comparison of the pacing phases revealed no significant differences. The deviations between the mean ITI and IOI were very small, but only for the IOI of 5,000 ms; the mean SD was significantly larger in tasks 2 and 3 than the self-paced condition (task 1 mean $\mathrm{SD}, 138.1 \mathrm{~ms})$.

The mean and SD of the ITI for task 1 and the continuation phase of tasks 2 and 3 are shown in Table 2. For an IOI of 1,000 ms, one-way ANOVA revealed a significant differ- 
Table 2. ITIs of task 1 and the continuation phases of tasks 2 and 3

\begin{tabular}{|c|c|c|c|c|c|c|}
\hline \multirow[b]{2}{*}{ IOI (ms) } & \multicolumn{3}{|c|}{ Mean (ms) } & \multicolumn{3}{|c|}{$\mathrm{SD}(\mathrm{ms})$} \\
\hline & task 1 & task 2 & task 3 & task 1 & task 2 & task 3 \\
\hline 1,000 & $1,137.0 \pm 202.9$ & $1,000.7 \pm 51.7 * *$ & $1,022.8 \pm 77.8^{*}$ & $45.3 \pm 17.6$ & $34.1 \pm 9.5$ & $40.3 \pm 17.1$ \\
\hline 2,000 & $1,847.9 \pm 66.3$ & $1,982.5 \pm 187.2$ & $2,077.6 \pm 325.5$ & $66.3 \pm 22.3$ & $81.5 \pm 36.1$ & $102.7 \pm 42.7^{* *}$ \\
\hline 5,000 & $4,177.8 \pm 1,050.8$ & $5,202.6 \pm 381.8^{* *}$ & $5,342.8 \pm 511.4^{* *}$ & $138.1 \pm 51.2$ & $282.1 \pm 100.3^{* *}$ & $320.9 \pm 166.1 * *$ \\
\hline
\end{tabular}

${ }^{*} \mathrm{p}<0.05$ compared with task $1,{ }^{* *} \mathrm{p}<0.01$ compared with task 1 , Values are mean \pm SD, IOI: inter-onset interval
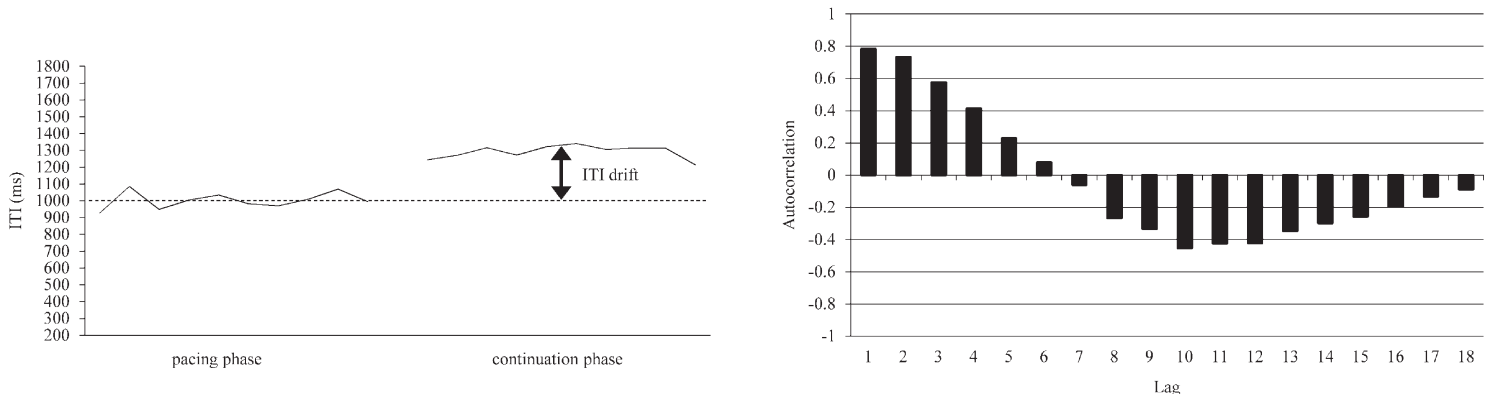

Fig. 3. Representative example of an ITI series with ITI drift in the continuation phase (left panel). Autocorrelation functions for the ITI time series consisted of 10 pacing-phase ITIs and 10 continuation-phase ITIs in this representative example (right panel).

ence between the mean ITI of the different tasks. Post-hoc analyses showed that the mean ITI was significantly smaller for task $2(\mathrm{p}<0.01)$ and task $3(\mathrm{p}<0.05)$ than for task 1. For an IOI of $2,000 \mathrm{~ms}$, the mean SD was significantly larger for task $3(p<0.01)$ than for task 1. For an IOI of 5,000 ms, the mean ITI was significantly larger for task $2(p<0.01)$ and task $3(\mathrm{p}<0.01)$ than for task 1 , and the mean SD was significantly larger for task $2(\mathrm{p}<0.01)$ and task $3(\mathrm{p}<0.01)$ than for task 1 . There were no significant differences in the other comparisons.

In the time series analysis of the continuation paradigm, the task 3 ITI $(1,000 \mathrm{~ms})$ was observed to drift in the continuation phase following the synchronization phase (Fig. 3 , left panel). For the time series data (10 pacing phase ITIs and 10 continuation phase ITIs) autocorrelation functions (correlogram) revealed certain trends. A positive peak appears in the lag 1 autocorrelation, and a negative peak appears in the lag 10 autocorrelation (Fig. 3, right panel). The appearance of a negative peak in the lag 10 autocorrelation shows that the ITI has a drift in the continuation phase. For tasks 2 and 3, the criteria for data selection were set as lag $1>0.4$ and/or lag 10 or lag $11<-0.4$. The trials that satisfied these criteria were selected. For task 2, there were 1, 6 and 4 subjects out of 18 who met the criteria for IOIs of 1,000 , 2,000 and 5,000 ms, respectively. For task 3, there were 1, 9 and 8 subjects out of 18 who met the criteria for IOIs of 1,000, 2,000 and 5,000 ms, respectively. The ITI series that were similar to the example shown in Fig. 3 clearly indicated drifts in the continuation phase.

\section{DISCUSSION}

Variability in ITIs during a finger-tapping task is an important indicator of a subject's rhythmic movement skill.
For self-paced tapping, finger tapping produces a series of intervals with substantial variability, even when they are intended to be regular; the typical SD is $3-6 \%$ of the IOI within a range of $200-2,000 \mathrm{~ms}^{5}$. In this study, the mean SD of the ITI for task 1 was $4.5 \%, 3.3 \%$ and $2.8 \%$ of the IOI for 1,000, 2,000 and 5,000 ms, respectively. There were individual differences in the degree of deviation between the target duration and the ITI. Each subject seems to have made an estimate of interval duration based on past experience. When they were asked to tap at a constant rhythm with a specific interval, the consistency of the movement took precedence over the accuracy of the time interval.

Rhythmic synchronization tasks such as those used in the pacing phase of this study require two timing demands: to produce a rhythm with the same frequency as an external stimulus and to produce motor responses that coincide in time with the stimulus or the midpoint of each stimulus. Although the sensorimotor asynchronies are clearly different between the synchronization and syncopation conditions, they are equally effective between in-phase tapping (synonymous with the synchronization tapping) and anti-phase tapping (synonymous with the syncopation tapping). The degree of anticipation of the IOI midpoints in anti-phase tapping was shown to be very similar to that of the tone onset in in-phase tapping ${ }^{11)}$. A study of syncopated rhythmic sensorimotor synchronization showed that adaptation of the response interval to a step change in the stimulus interval was almost immediate, but there was no such adaptation in synchronization error $(\mathrm{SE})^{12}$. Complete compensation for subliminal pulse changes has been reported to occur within 2 or 3 taps when the taps are intended to be in phase with the tones ${ }^{11)}$. These findings suggest that frequency is the most important timing demand. In the pacing phase of this study, there was no difference in the variability of tapping 
between the synchronization and syncopation conditions. The rhythmicity of periodic movement may have been controlled mainly on the basis of frequency correction rather than the asynchronies. However, it should be noted that the findings of previous studies were based on intervals of less than $1,000 \mathrm{~ms}$.

In the pacing phase of this study, several subjects developed wavy ITI series as the duration of the IOI increased. The rate at which listeners are most likely to infer tempi from basic pulse rates ranges from 60 to 150 beats per minute $(\mathrm{bpm})^{13)}$. This study used 60, 30, and $12 \mathrm{bpm}$ for IOIs of $1,000,2,000$ and $5,000 \mathrm{~ms}$, respectively. For IOIs of 2,000 and $5,000 \mathrm{~ms}$, it was not easy for the subjects to judge the tempo, and stimuli may have been felt as mere pulse repetitions. A study using synchronization tapping in parallel with a word-memory task found that the reduced attention due to the execution of the secondary task did not have a significant effect on automatic anticipation of IOIs in the range of $450-1,500 \mathrm{~ms}^{14)}$. However, for IOIs in the range of $1,800-3,600 \mathrm{~ms}$, anticipatory tapping was substantially affected by tasks that decreased attentional resources. These results suggest that a timing-control mechanism independent of attentional resources, a so-called automatic movement, exists for IOIs below $1,800 \mathrm{~ms}$ and that tapping is controlled in a feed-forward rather than a feed-back manner.

For periodic movement associated with a feed-back manner, sensorimotor asynchrony seems to provide information that influences the timing of the next tap. In fact, in this study, the distribution of SE of the IOI of $1,000 \mathrm{~ms}$ showed a similar tendency to that reported in a previous study in which the SE almost always indicated negative asynchrony, with a small spread in SE distribution for short IOIs $(450-1,500 \mathrm{~ms})^{14)}$. In this study, we observed that as the duration of the IOI increased, the peak distribution decreased. In particular, negative asynchrony and positive asynchrony were intermingled, and the SE distribution was broad for an IOI of 5,000 ms under both conditions. Because several subjects controlled movement based on asynchronies, there were deviations between ITI and the target duration in the pacing phase. Under both the synchronization and syncopation conditions, periodic movement could be performed as an automatic movement for the 1,000 ms IOI. Thus, even after pacing was removed, the continuation of automatic periodic movement was easily performed by the subjects. The subsequent mean ITI in the continuation phase was closer to $1,000 \mathrm{~ms}$ than that of self-paced tapping. Although this observation may be specific to tasks with an IOI of $1,000 \mathrm{~ms}$, autocorrelation functions in tasks 2 and 3 revealed trends similar to that shown in Fig. 3, with ITI drift during each task. The mean ITI in the continuation phase was 1,169.5 ms (task 2) and 1,291 ms (task 3), and the drift interval was longer than $1,000 \mathrm{~ms}$. Furthermore, the mean ITI under the self-paced condition of one subject was 1,387.9 ms (task 2) and 1,429.9 ms (task 3). The deviation between the mean ITI and 1,000 ms was positive. Although it is not difficult to perform automatic movement with an interval of $1,000 \mathrm{~ms}$, if the deviation between the ITI and $1,000 \mathrm{~ms}$ is large under self-paced conditions, the effects of pacing may become too small for adaptation to the interval.

For an IOI of 2,000 ms, comparison of task 1 with the continuation phase of tasks 2 and 3 showed the mean SD was significantly high under self-paced conditions, but only for the syncopation component. The ITI series of task 3 for an IOI of 2,000 ms (Fig. 2) indicates that several subjects drifted away from the 2,000 ms interval during the continuation phase, even though the mean ITI for the 18 subjects was 2,077.6 ms. Based on results of autocorrelation functions for ITIs, the 18 subjects were divided into 2 groups: 9 subjects in the ITI drift group and 9 subjects in the ITI nodrift group. Additional analysis using the unpaired t-test revealed no significant differences between the groups during the pacing (syncopation) phase. In the continuation phase, the mean SD of the drift group was significantly higher than that of the no-drift group. We observed a large variation in the ITI during the continuation phase among the subjects with ITI drift. The interval of $2,000 \mathrm{~ms}$ was close to the $1,800 \mathrm{~ms}$ interval reported by Miyake et al. ${ }^{14}$ ), and the difficulty of periodic movement with a 2,000 ms interval was different for each individual.

One of the strategies used to perform syncopation tapping with a 2,000 ms interval involves a stimulus and tap repeated at intervals of $1,000 \mathrm{~ms}$, halfway through the $2,000 \mathrm{~ms}$ interval. This strategy, which cannot be used in synchronization tapping, has been adopted in syncopation tapping. For the execution of this strategy, accurate estimation of the $1,000 \mathrm{~ms}$ interval is required. Further, additional analysis demonstrated that the correlation coefficient is 0.42 for the mean ITI between task $1(1,000 \mathrm{~ms})$ and the continuation phase of task $3(2,000 \mathrm{~ms})$. In the continuation phase following the syncopation tapping, the subjects had to estimate a 1,000 ms interval on their own to determine the time between the lack of stimulus and their tap. However, since deviations from the target duration were large during the self-paced tapping at the $1,000 \mathrm{~ms}$ interval (i.e., the ability to estimate a $1,000 \mathrm{~ms}$ interval was poor), accurate movement in the continuation phase would be more difficult. If the syncopation tapping of the interval of $2,000 \mathrm{~ms}$ were adopted as pacing, the subjects should be able to accurately estimate the halfway point of the time interval.

For an IOI of 5,000 ms, we classified the SE of synchronization tapping into 2 types: anticipatory tapping, in which negative asynchrony is observed, and reactive tapping to a stimulus. Anticipatory tapping and reactive tapping were identified when the standard value of SE was set to $100 \mathrm{~ms}^{14}$ ). When we adopted this criterion, the SE data for task 2 identified reactive tapping $(0,13$, and 52 out of 180 SE data points for IOI 1,000, 2,000 and 5,000 ms, respectively). Reactive tapping occurs after the identification of a stimulus, such that predicting when the next stimulus will occur is difficult. For IOIs longer than $5,000 \mathrm{~ms}$, estimation of temporal duration was shown to involve memory ${ }^{15}$, which concurs with the finding in our study that reactive tapping markedly increased with an IOI of 5,000 ms. In addition, the histogram of SE distribution was flat and wide, and did not demonstrate a normal distribution. These findings showed the $5,000 \mathrm{~ms}$ task is performed in a non-rhythmical manner and relies on memory more than timing. True 
rhythm should refer only to events within the time scale of short-term memory ${ }^{13)}$. Intervals of $5,000 \mathrm{~ms}$ are probably too long for facilitation of movement using pacing, as movement under both the synchronization and syncopation conditions would be controlled by memory; thus, variability in the periodic movement would be large. Even though the ITI of the self-paced condition deviated considerably from the actual 5,000 ms interval, the consistency of the tapping was maintained. Despite individual differences, the variability probably represents the maximum performance for finger tapping.

Clinical studies have reported that intervention using rhythmic auditory stimulation improved gait velocity, cadence, and stride length of patients with Parkinson's disease ${ }^{16)}$, and gait velocity, stride length, and electromyographic activity of the medial gastrocnemius of hemiparetic stroke patients ${ }^{17)}$. Our investigation may have clinical applications, as we demonstrated that pacing is effective when the IOI is shorter than $1,000 \mathrm{~ms}$. The average cadence of healthy subjects is $110 \mathrm{steps} / \mathrm{s}^{18)}$, which corresponds to an IOI of about $545 \mathrm{~ms}$. Our finding that the optimal pacing signal interval is $1,000 \mathrm{~ms}$ may help in the cuing of the sequence of events that occur between successive heel contacts of the same foot. However, if the average time of a gait cycle were close to the target duration, cuing by the pacing signal would be unnecessary. Pacing at an interval of $1,000 \mathrm{~ms}$ would be useful for determining the correct gait speed and step rate during gait training. During physical therapy, the repetition of slow, intentionally performed movements is often used to train eccentric contraction of the muscles involved in the movements. For example, when the task of sitting down from a standing position is performed more slowly, eccentric contraction of the quadriceps is required. If such slow movements were repeated at 5-second intervals, external stimuli and verbal instructions would be necessary to insert a secondary event every $1,000 \mathrm{~ms}$.

A limitation of this study was that only the instant effect of pacing was examined using the continuation paradigm. In particular, for the 5,000 and 2,000 ms tasks, in the future, it will be necessary to evaluate the consistency of periodic movement to examine not only the instant effect of pacing but also the training effect of longitudinal intervention.

\section{REFERENCES}

1) Safranek MG, Koshland GF, Raymond G: Effect of auditory rhythm on muscle activity. Phys Ther, 1982, 62: 161-168. [Medline]

2) Del Olmo MF, Cheeran B, Koch G, et al.: Role of the cerebellum in externally paced rhythmic finger movement. J Neurophysiol, 2007, 98: 145-152. [Medline] [CrossRef]

3) Lewis PA, Wing AM, Pope PA, et al.: Brain activity correlates differentially with increasing temporal complexity of rhythms during initialization, synchronization, and continuation phases of paced finger tapping. Neuropsychologia, 2004, 42: 1301-1312. [Medline] [CrossRef]

4) Rao SM, Harrington DL, Haaland KY, et al.: Distributed neural systems underlying the timing of movements. J Neurosci, 1997, 17: 5528-5535. [Medline]

5) Madison G: Variability in isochronous tapping: higher order dependencies as a function of intertap interval. J Exp Psychol Hum Percept Perform, 2001, 27: 411-422. [Medline] [CrossRef]

6) Semjen A, Schulze HH, Vorberg D: Timing precision in continuation and synchronization tapping. Psychol Res, 2000, 63: 137-147. [Medline] [CrossRef]

7) Oullier O, Jantzen KJ, Steinberg FL, et al.: Neural substrates of real and imagined sensorimotor coordination. Cereb Cortex, 2005, 15: 975-985. [Medline] [CrossRef]

8) Jantzen KJ, Oullier O, Marshall M, et al.: A parametric fMRI investigation of context effects in sensorimotor timing and coordination. Neuropsychologia, 2007, 45: 673-684. [Medline] [CrossRef]

9) Engström DA, Kelso JA, Holroyd T: Reaction-anticipation transitions in human perception-action patterns. Hum Mov Sci, 1996, 15: 809-832. [CrossRef]

10) Oldfield RC: The assessment and analysis of handedness: the Edinburgh inventory. Neuropsychologia, 1971, 9: 97-113. [Medline] [CrossRef]

11) Repp BH: Phase correction, phase resetting, and phase shifts after subliminal timing perturbations in sensorimotor synchronization. J Exp Psychol Hum Percept Perform, 2001, 27: 600-621. [Medline] [CrossRef]

12) Thaut MH, Kenyon GP: Rapid motor adaptations to subliminal frequency shifts during syncopated rhythmic sensorimotor synchronization. Hum Mov Sci, 2003, 22: 321-338. [Medline] [CrossRef]

13) Thaut MH: Rhythm, music, and the brain: scientific foundations and clinical applications. New York: Routledge, 2008, pp 6-15.

14) Miyake Y, Onishi Y, Pöppel E: Two types of anticipation in synchronization tapping. Acta Neurobiol Exp (Warsz), 2004, 64: 415-426. [Medline]

15) Fraisse P: Perception and estimation of time. Annu Rev Psychol, 1984, 35: 1-36. [Medline] [CrossRef]

16) McIntosh GC, Brown SH, Rice RR, et al.: Rhythmic auditory-motor facilitation of gait patterns in patients with Parkinson's disease. J Neurol Neurosurg Psychiatry, 1997, 62: 22-26. [Medline] [CrossRef]

17) Thaut MH, McIntosh GC, Rice RR: Rhythmic facilitation of gait training in hemiparetic stroke rehabilitation. J Neurol Sci, 1997, 151: 207-212. [Medline] [CrossRef]

18) Neumann DA: Kinesiology of the musculoskeletal system: foundations for physical rehabilitation. St. Louis: Mosby, 2002, pp 527-529. 